\title{
Postoperative Neovascularization, Cerebral Hemodynamics and Clinical Prognosis Between Combined and Indirect bypass Revascularization Procedures in Hemorrhagic Moyamoya Disease.
}

\section{Jun Sun}

Third Affiliated Hospital of Sun Yat-Sen University https://orcid.org/0000-0003-4346-6313

\section{Yu Zhang Li}

Third Affiliated Hospital of Sun Yat-Sen University

\section{Chuan Chen}

Third Affiliated Hospital of Sun Yat-Sen University

\section{Cong Ling}

Third Affiliated Hospital of Sun Yat-Sen University

Hao Li

Third Affiliated Hospital of Sun Yat-Sen University

Hui Wang ( $\nabla$ doctorwanghui@126.com )

\section{Research article}

Keywords: Hemorrhagic MMD, Combined and indirect revascularization, Hemodynamics, Postoperative collateral formation, Clinical prognosis

Posted Date: July 24th, 2020

DOI: https://doi.org/10.21203/rs.3.rs-43687/v1

License: (c) (i) This work is licensed under a Creative Commons Attribution 4.0 International License. Read Full License

Version of Record: A version of this preprint was published at Clinical Neurology and Neurosurgery on August 1st, 2021. See the published version at https://doi.org/10.1016/j.clineuro.2021.106869. 


\section{Abstract}

BACKGROUND: Present primary focus was rebleeding and prognosis of hemorrhagic moyamoya disease (MMD), while limited researches emphasized the postoperative collateral formation and cerebral hemodynamics.

OBJECTIVE: To compare the diverse effects of combined (CB) and indirect (IB) bypass revascularization procedures on the postoperative collateral formation and hemodynamics in hemorrhagic MMD patients.

METHODS: Hemorrhagic MMD patients treated surgically were retrospectively collected and dichotomized into CB and IB group. Postoperative complications and clinical prognosis, as well as preand post-operative Modified Rankin Scale (mRS), collateral circulation status and cerebral hemodynamics were observed and compared between the CB and IB groups.

RESULTS: A total of 37 patients with hemorrhagic MMD were identified. Of 68 cerebral hemispheres, $47(69.1 \%)$ were combined revascularization and the rest indirect. During an average follow-up of 16.5 \pm 8.7 months, the recurrent stroke events were significantly lower, as well as postoperative mRS scores $\leq 2$, satisfactory postoperative collateral formation, improved dilation or extension of anterior choroidal /posterior communication artery (AchA/PcoA) were significantly higher in the CB group than in the IB group (all $P<$.05). Compared with preoperative cerebral hemodynamics, relative cerebral blood flow (rCBF), relative cerebral blood volume (rCBV), mean transit time (MTT) and relative time to peak (rTTP) in the $\mathrm{CB}$ group; but in the IB group, $\mathrm{rCBF}$, rCBV and MTT were significantly improved (all $P<.001$ ). The $\mathrm{CB}$ group's postoperative rCBF was significantly improved as compared with the IB group $(P<.001)$.

CONCLUSION: CB can obtain better postoperative collateral formation, cerebral hemodynamics, and clinical prognosis as compared with IB.

\section{Introduction:}

Moyamoya disease (MMD) is a chronic, progressive and irreversible cerebral vasculopathy which is characterized by progressive stenosis or occlusion of the bilateral or unilateral distal intracranial internal carotid arteries, and the proximal anterior cerebral artery and middle cerebral arteries, with abnormal moyamoya vessels formation at the skull bases. ${ }^{1-2} \mathrm{MMD}$ usually exhibits ischemic or hemorrhagic stroke and most occurs in 0 to 10 or 30 to 50 -year-old patients. ${ }^{3-4}$ The ischemic MMD mainly occurs in pediatric populations, while the hemorrhagic MMD usually occurs in adults and has higher mortality and disability. ${ }^{4-8}$ Recently, many studies including the Japanese adult moyamoya disease trial have shown that compared with conservative treatment, surgical revascularization can significantly reduce the rehemorrhage in patients with hemorrhagic MMD, but there has been a debate on the optimal surgical procedure. ${ }^{5,9-16}$ For example, Deng $X$ et al. have reported that in patients with hemorrhagic MMD, combined (CB), directed (DB) and indirect (IB) revascularization surgery has no significant difference in

preventing rebleeding, while Park SE et al. believed that CB and DB were more effective than IB. ${ }^{12,15}$ Even, 
Macyszyn et al.'s meta-analysis found that IB and CB were superior to DB. ${ }^{17}$ Despite the controversy, most investigators believe that $\mathrm{CB}$ is better than IB. Most of the present studies focused on the analyses of postoperative clinical prognosis, rehemorrhage rate and related risk factors, while few highlighted simultaneously the neovascularization and cerebral hemodynamics, which the study conducted.

\section{Methods:}

This study was approved by the Biomedical Ethics Committee of our hospital. Informed consent was obtained from all patients.

\section{Study Population:}

We retrospectively collected the demographic information, underlying diseases, clinical and radiographic information of 37 patients with hemorrhagic MMD admitted in our hospital's department of neurosurgery from March 2014 to August 2019 in this cohort study. According to the different surgical procedures, they were dichotomized into the combined surgical revascularization (CB) and the indirect (IB) groups.

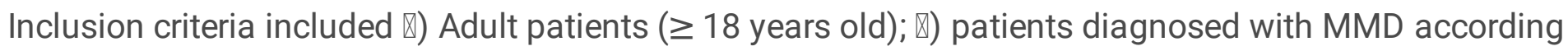
to the guidelines made by the Research Committee on MMD in Japan; $\left.{ }^{23} \rrbracket\right)$ intracranial hemorrhage occurred in the last $1 \otimes 12$ months and $\rrbracket$ ) received CB or IB surgery;

Exclusion criteria were that $₫$ ) Patients diagnosed with moyamoya syndrome whose cause has been

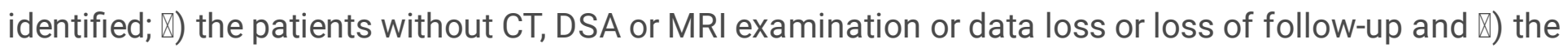
patients rejected revascularization surgery or had $\mathrm{mRS}$ score $\geq 5$ points and were unsuitable for followup.

\section{Surgical Technique:}

Both $\mathrm{CB}$ and IB are performed by the neurosurgeons with at least 10-year-experience in cerebrovascular surgery. $C B$ was used when the patient has appropriate recipient and donor arteries, and or else, IB was used. $\mathrm{CB}$ selected superficial temporal artery-to-middle cerebral artery anastomosis combined with encephaloduroarteriomyosynangiosis (STA-MCA + EDAMS), and IB selected encephaloduroarteriomyosynangiosis (EDAMS). All patients underwent CTA and CTP examination on the first day after surgery to check for postoperative hemorrhage or infarction and perfusion.

\section{Radiological and Clinical Evaluation}

We control the patients' perioperative blood pressure under $140 / 100 \mathrm{mmHg}$ and blood glucose under $7.1 \mathrm{mmol} / \mathrm{L}$. Antiplatelet therapy was not administrated because of increasing the risk of postoperative rebleeding. Patients were followed up 3-6 months after surgery and every year. The pre-/post-operative complications, stroke events, DSA, CTP and mRS score were recorded. 
According to clinical manifestations, we evaluated pre- and post-operative neurological function with modified Rankin scale(mRS). All patients underwent pre- and post-operative DSA and CTP examination. According to DSA, we evaluated the preoperative collateral circulation based on the classification criteria proposed by Liu et al. (Table 1, Fig. 1), the neovascularization based on Matsushima scale (Table 2), the regression of moyamoya vessels and the improvement of the AchA/PcoA dilation or extension. ${ }^{18,19}$ According to the CTP, we assessed the preoperative cerebral hemodynamics based on CTP stage proposed by Yin et al. (Table 3), and recorded the pre- and post-operative parameters including relative cerebral blood flow (rCBF), relative cerebral blood volume ( $\mathrm{rCBV}$ ), relative time to peak (rTTP) and mean transit time (MTT). ${ }^{20}$ According to the studies of Chung Jung Lin et al. and M. Wintermark et al, MTT has a better correlation between CTP and magnetic resonance perfusion imaging than $\mathrm{rMTT}$ in ischemic encephalopathy, so we chose MTT in this study. ${ }^{21,22}$ We separately evaluated each hemisphere for every individual. 
Table 1

Collateral circulation grading system proposed by Liu ZW et al. ${ }^{20}$

Collateral circulation

Score

Basal brain perforators and moyamoya vessels

\begin{tabular}{llr}
$\begin{array}{l}\text { Suzuki } \\
\text { stage }\end{array}$ & Stage 1 & 6 \\
\cline { 2 - 3 } & Stage 2 & 5 \\
\hline Stage 3 & 3 \\
\hline Stage 4 & 2 \\
\hline Stage 5 & 1 \\
\hline Stage 6 &
\end{tabular}

The leptomeningeal system

Normal or stenosed posterior cerebral artery (PCA) without leptomeningeal $\quad 0$ anastomoses.

$\mathrm{pPCA} \rightarrow \quad$ Parietoccipital branches of the PCA $\rightarrow$ cortical border zone between the ACA $\quad 1$ ACA and PCA.

Parietoccipital branches of the PCA and posterior pericallosal $\rightarrow$ ACA territory $\quad 2$ over the Central Sulcus.

tPCA $\rightarrow \quad$ Temporal PCA branches $\rightarrow$ Temporal MCA territory.

tMCA

$\mathrm{pPCA} \rightarrow \quad$ Parietoccipital PCA branches $\rightarrow$ only in the superficial vessels (M4 segment) of

MCA the MCA.

Parietoccipital PCA branches $\rightarrow$ extending into the sylvian fissure (M3 segment) of the MCA.

Parietoccipital PCA branches $\rightarrow$ up to the occlusion (M1 or M2 segment) of the 3 MCA.

\section{Grade}

$\begin{array}{ll}\square & 1 \rrbracket 4 \\ \square & 5 \rrbracket 8 \\ \square & 9 \rrbracket 12\end{array}$

Abbreviations: $M C A$ middle cerebral artery, $P C A$ posterior cerebral artery, $P P C A$ Parietoccipital branches of the posterior cerebral artery, $t P C A$ Temporal posterior cerebral artery-branches, $A C A$ anterior cerebral artery. 
Table 2

Matsushima Scale ${ }^{21}$

\begin{tabular}{|ll|}
\hline Grade & Characters \\
\hline A & As the revascularization of $>2 / 3$ of the MCA distribution \\
\hline B & As the revascularization of $1 / 3 \sim 2 / 3$ of the MCA distribution \\
\hline C & As the revascularization of $<1 / 3$ of the MCA distribution or none \\
\hline Abbreviations: $M C A$ middle cerebral artery. \\
\hline
\end{tabular}

Table 3

The Stage of CTP proposed by Yin et al. ${ }^{22}$

\begin{tabular}{|ll|}
\hline Stage & Characters \\
\hline $\begin{array}{l}\text { Normal } \\
\square\end{array}$ & - \\
\hline$\square$ & TTP was delayed, MTT, rCBF and rCBV were normal \\
& normal or slightly increased \\
\hline$\square$ & $\begin{array}{l}\text { TTP and MTT were delayed, rCBF was decreased, and rCBV was normal or slightly } \\
\text { decreased }\end{array}$ \\
\hline$\square$ & TTP and MTT were delayed, rCBF and rCBV were decreased \\
\hline $\begin{array}{l}\text { Abbreviations: } M T T \text { mean transit time, } T T P \text { time to peak, } r C B F \text { relative indicates relative cerebral } \\
\text { blood flow, rCBV relative cerebral blood volume; }\end{array}$ \\
\hline
\end{tabular}

\section{Statistical Analysis:}

All statistical analyses were carried out using SPSS software (Windows version 22.0; IBM). The categorical variables and the continuous variables were represented by percentiles and mean \pm standard deviation respectively. Independent sample t-test was used for measurement variables such as age, and chi-square test or paired rank-sum test was used for classification variables such as gender, postoperative complications, pre- and post-operative collateral circulation grading, and mRS score. $P<.05$ indicates statistical significance.

\section{Results:}

\section{Patient Characteristics:}

37 patients with hemorrhagic MMD, and 68 hemispheres underwent surgical revascularization was identified. The age and gender were no statistical significance between the two groups $(P=.333, P=.215$, respectively). Preoperative clinical and radiological characteristics (the underlying diseases, bleeding site, 
collateral circulation, CTP stage, follow-up time and interval from hemorrhage to revascularization) were not significant difference (Table 4). 
Table 4

Baseline characteristics

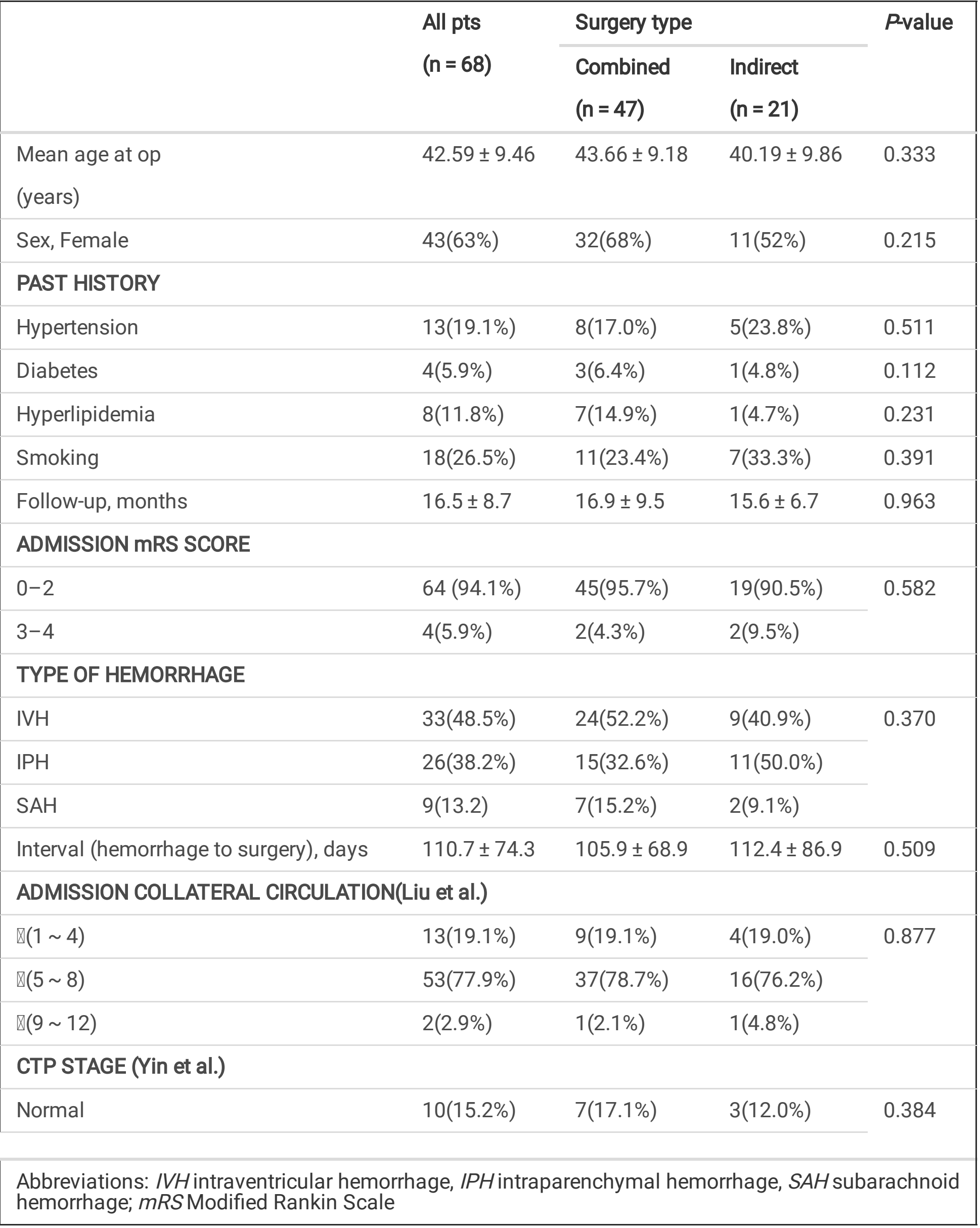




\begin{tabular}{|c|c|c|c|c|}
\hline & \multirow{3}{*}{$\begin{array}{l}\text { All pts } \\
(n=68)\end{array}$} & \multicolumn{2}{|c|}{ Surgery type } & \multirow[t]{3}{*}{$P$-value } \\
\hline & & Combined & Indirect & \\
\hline & & $(n=47)$ & $(n=21)$ & \\
\hline ? & $2(3.0 \%)$ & $2(4.9 \%)$ & $0(0.0 \%)$ & \\
\hline ( & $14(21.2 \%)$ & $6(14.6 \%)$ & $8(32.0 \%)$ & \\
\hline [ & $19(28.8 \%)$ & $11(26.8 \%)$ & $8(32.0 \%)$ & \\
\hline ૧ & $21(31.8 \%)$ & $15(36.6 \%)$ & $6(24.0 \%)$ & \\
\hline
\end{tabular}

\section{Postoperative complications and prognosis:}

Over the average $16.5 \pm 8.7$ months follow-up duration, in the CB group, recurrent hemorrhagic stroke occurred in 1 of 47 hemispheres $(2.1 \%)$ and no ischemic stroke occurred $(0.0 \%)$. In the IB group, recurrent hemorrhagic stroke occurred in 4 of 21 hemispheres (19.0\%) and ischemic stroke occurred in 3 of 21 hemispheres (14.2\%). Recurrent hemorrhagic and ischemic stroke were significantly different between the two groups ( $P=.029$ and $P=.027$, respectively), while postoperative delayed wound healing was statistical significance $(P=.309)$. The patients with $\mathrm{mRS} \leq 2$ points in the $\mathrm{CB}$ group were more demonstrable $(P=.009)$ (Table 5).

Table 5

Postoperative complications and outcome

\begin{tabular}{|c|c|c|c|c|}
\hline & \multirow{2}{*}{$\begin{array}{l}\text { All pts } \\
(n=68)\end{array}$} & \multicolumn{2}{|c|}{ Surgery type } & \multirow[t]{2}{*}{$P$-value } \\
\hline & & $\begin{array}{l}\text { Combined } \\
(n=47)\end{array}$ & $\begin{array}{l}\text { Indirect } \\
(n=21)\end{array}$ & \\
\hline \multicolumn{5}{|c|}{ POSTOPERATIVE COMPLICATIONS } \\
\hline Ischemic events & $3(4.4 \%)$ & $0(0.0 \%)$ & $3(14.2 \%)$ & $0.027^{*}$ \\
\hline Rebleeding & $5(7.4 \%)$ & $1(2.1 \%)$ & $4(19.0 \%)$ & $0.029^{*}$ \\
\hline Delayed wound healing & $1(1.5 \%)$ & $0(0.0 \%)$ & $1(4.8 \%)$ & 0.309 \\
\hline \multicolumn{5}{|l|}{ mRS SCORE } \\
\hline $0-2$ & $62(91.2 \%)$ & $46(97.9 \%)$ & $16(76.2 \%)$ & \multirow[t]{2}{*}{$0.009^{*}$} \\
\hline $3-5$ & $6(8.8 \%)$ & $1(2.1 \%)$ & $5(23.8 \%)$ & \\
\hline
\end{tabular}

\section{Postoperative collateral circulation:}


According to the latterly postoperative DSA, we assessed the neovascularization based on the criteria of Matsushima scale. In the CB group, 41 of 47 hemispheres (87.2\%) obtained satisfied (Good, grade A and $B)$ neovascularization formation, and 11 of 21 hemispheres (52.4\%) in the IB group, with significant difference between the two groups $(P=.03)$. In the $\mathrm{CB}$ group, AchA/PcoA dilation or extension were improved in 19 of 47 hemispheres $(40.4 \%)$, and in 2 of 21 hemispheres $(9.5 \%)$ in the IB group, with a significant difference between the two groups $(P=.011)$. However, there was no significant difference in the reduction of moyamoya vessels $(P=.762)$ (Table 6$)$.

Table 6

Postoperative collateral circulation

\begin{tabular}{|c|c|c|c|c|}
\hline & \multirow{2}{*}{$\begin{array}{l}\text { All pts } \\
(n=68)\end{array}$} & \multicolumn{2}{|c|}{ Surgery type } & \multirow[t]{2}{*}{$P$-value } \\
\hline & & $\begin{array}{l}\text { Combined } \\
(n=47)\end{array}$ & $\begin{array}{l}\text { Indirect } \\
(n=21)\end{array}$ & \\
\hline \multicolumn{5}{|l|}{ Matsushima score } \\
\hline Good (grade A and B) & $52(76.5 \%)$ & $41(87.2 \%)$ & $11(52.4 \%)$ & \multirow[t]{2}{*}{$0.003^{*}$} \\
\hline Poor (grade C) & $16(23.5 \%)$ & $6(12.8 \%)$ & $10(47.6 \%)$ & \\
\hline $\begin{array}{l}\text { Improvement of AchA/PcoA dilation } \\
\text { or extension }\end{array}$ & $22(32.4 \%)$ & $19(40.4 \%)$ & $2(9.5 \%)$ & $0.011^{*}$ \\
\hline $\begin{array}{l}\text { Decrease of moyamoya } \\
\text { vessels }\end{array}$ & $31(45.6 \%)$ & $22(46.8 \%)$ & $9(43.9 \%)$ & 0.762 \\
\hline \multicolumn{5}{|l|}{${ }^{*} P<0.05$ means statistical significance; } \\
\hline \multicolumn{5}{|c|}{ Abbreviations: $A c h A / P c o A$ the anterior choroid artery and posterior communication artery } \\
\hline
\end{tabular}

According to the latest CTP, in the CB group, postoperative rCBF, rCBV, MTT and rTTP were significantly improved as compared with the preoperative ones (all $P<.001$ ). In the IB group, postoperative rCBF, rCBV and MTT were significantly improved as compared with those before surgery $(P<.001)$ except rTTP $(P$ $>$.05). Only the improvement of postoperative rCBF in the CB group was statistically different as compared with that of the IB group $(P<.001)$ (Table 7). 
Table 7

Preoperative and postoperative CTP parameters

Surgery type

Combined

$(n=47)$
$P$-value

Indirect

$(n=21)$

\section{rCBF}

Preoperative

$0.71 \pm 0.13$

$0.73 \pm 0.13$

0.686

Postoperative

$1.65 \pm 0.52$

$1.15 \pm 0.12$

$<0.001^{*}$

P-value

$<0.001^{*}$

$<0.001^{*}$

rCBV

\begin{tabular}{llll} 
Preoperative & $0.84 \pm 0.15$ & $0.89 \pm 0.10$ & 0.878 \\
Postoperative & $1.56 \pm 0.56$ & $1.18 \pm 0.15$ & 0.868 \\
\hline P-value & $<0.001^{*}$ & $<0.001^{*}$ &
\end{tabular}

MTT

Preoperative

$5.36 \pm 1.03$

$4.98 \pm 0.84$

0.091

Postoperative

$4.16 \pm 1.22$

$3.92 \pm 0.72$

0.388

P-value

$<0.001^{*}$

$<0.001^{*}$

rTTP

Preoperative

$1.12 \pm 0.07$

$1.06 \pm 0.04$

0.847

Postoperative

$0.98 \pm 0.12$

$0.95 \pm 0.10$

0.353

P-value

$<0.001^{*}$

0.067

${ }^{*} P<0.05$ means significant difference;

Abbreviations: $r C B F$ relative cerebral blood flow, $r C B V$ relative cerebral blood volume, $M T T$ mean transit time, $r T T P$ relative time to peak. We set the area of cerebellum as the reference.

\section{Case Illustration:}

Case-1: A 43-year-old male patient without hypertension, diabetes and hyperlipidemia underwent CB (combined STA-MCA with EDAMS surgery) in the left hemisphere due to a hemorrhage in left basal ganglia. His preoperative collateral status was assessed as 8 scores, and the preoperative stage of CTP

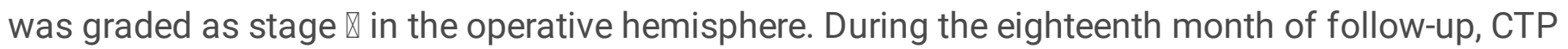
indicated improved TTP and CBV, but did not CBF and MTT at the temporal region, and DSA showed 
satisfactory revascularization (Matsushima grade A) and the improved AchA-PcoA dilation and extension. This patient had also improved symptoms and neurofunctional state during the follow-up. CTP and DSA images are showed in Fig. 2.

Case-2: A 46-year-old female patient without hypertension, diabetes and hyperlipidemia underwent IB (EDAMS surgery) in the right hemisphere due to a hemorrhage in right temporal lobe. Her preoperative collateral status was assessed as 6 scores, and the preoperative stage of CTP was graded as stage $\nabla$ in the operative hemisphere. During the 16-month follow-up, DSA showed the satisfactory revascularization (Matsushima grade A) and the improved AchA-PcoA dilation and extension. CTP demonstrated improved overall parameters of cerebral perfusion. The symptoms of this patient were also improved during the follow-up. CTP and DSA images are showed in Fig. 3.

\section{Discussion:}

\section{Postoperative complication and clinical outcome}

Hemorrhagic MMD has high rehemorrhage rates between $32 \%$ and $61 \%$, usually leading to high mortality and disability. ${ }^{6-8,11}$ The primary emphasis has been to reduce this with a potential revascularization surgery rather than conservative treatment. Recent studies identified that surgical revascularization can significantly reduce the rehemorrhage rates to $12-17 \%$ and improves the clinical neurological function as compared with conservative treatment in patients with hemorrhagic MMD. 5, ${ }^{5,15,23}$ Although the optimal revascularization procedure among combined revascularization (CB), direct revascularization (DB) and indirect revascularization (IB) for hemorrhagic MMD is still controversial, most investigators believe that $\mathrm{CB}$ is superior to IB in preventing stroke recurrence, which was consistent with this study. This study also showed that the patients with $\mathrm{mRS} \leq 2$ points was significantly greater in the $\mathrm{CB}$ group than in the IB group ( $P=.009$ ). Therefore, compared with IB, $\mathrm{CB}$ can better reduce the incidence of recurrent stroke, avoid secondary damage to neurological function caused by rebleeding, and obtain better clinical prognosis. Since the electrocoagulation was rarely used during the operation as well as the temporal muscle and skin had rich blood supply, the postoperative delayed wound healing was no significantly different between the two groups $(P=.309)$.

At present, most studies have emphasized on the clinical prognosis, stroke recurrence and related risk factors, while few studies focus on why different revascularization procedures often company with various neurofunctional prognosis, and whether the diverse impacts on neovascularization and hemodynamics play a role. Therefore, we conduct this study to investigate the postoperative collateral circulation formation and cerebral hemodynamics between $\mathrm{CB}$ and IB, and to provide a foundation for improving the revascularization procedures and a guidance for utilizing the vasoactive and angiogenic medications.

\section{Postoperative collateral circulation}


Suzuki stage, a time-oriented classification, has been applied wildly to evaluate the progression of MMD, but it can't completely take the individualized level of collateral circulation into account. ${ }^{24-26}$ Thus, we adopted the novel classification criteria, proposed by Liu et al. (Table 1, Fig. 1) to assess the preoperative collateral circulation status in order to ensure the preoperative homogeneity between the two groups. ${ }^{18}$

In this study, we found that $\mathrm{CB}$ group had better neovascularization than IB group $(P=.03)$. It may be that the neovascularization of CB consisted of directed STA-MCA bypass vessels and sticking vessels including the meningeal arteries and deep temporal artery, etc, while the IB group only possessed the sticking vessels. Previous studies thought that DB can provide immediately the cerebral blood flow and promote neoangiogenesis, while IB will take at least 3 months to induce neovascularization with increased cerebral perfusion, but the long-term and stable effect on neovascularization should not be neglected. ${ }^{14-15,17,25,27}$ Although Park SE et al. and Zhao Y et al. believed that the role of indirect revascularization in promoting neovascularization was limited, a large proportion (52.4\%) of patients in our study obtained good neovascularization in the IB group. ${ }^{15,28}$ We hypothesis that the younger age of our patients and different procedures of IB might play a role. Peicong $\mathrm{Ge}$ et al. believed that the younger the patients at the operation, the better postoperative collateral formation. ${ }^{29}$ In addition, in their studies, encephaloduroarteriosynangiosis (EDAS) and encephalodurosynangiosis (EDS) procedures was performed, while we applied encephaloduroarteriomyosynangiosis (EDAMS) procedure, in which temporal muscle flap with deep temporal artery might be superior to EDS or EDAS in the ingrowth of new vessels. This is also consistent with the study of Zhao $\mathrm{J}$ et al. that the encephaloduromyosynangiosis (EDMS) operation can provide lasting revascularization. ${ }^{14}$ Meanwhile, the improvement of AchA-PcoA dilation or extension was significantly obvious in the CB group than in the IB group $(P=.011)$. We speculated that both the directed STA-MCA anastomosis and sticking vessels in CB group could reduce the hemodynamic stress of the terminal internal carotid artery and its abnormal branches in short-term or long-term, and improve the extension and dilation of AchA-PcoA, which was also the reason that the rehemorrhage rate is lower than the IB group. ${ }^{10,11,30}$ However, the regression of moyamoya vessels occurred in both CB and IB group, but there was no statistical significance between the two groups $(P$ $=.76$ ). We speculated that it might been caused by the not enough sample size and follow-up duration.

\section{Postoperative improvement of hemodynamics}

The postoperative rCBF, rCBV, MTT and rTTP were all significantly improved as compared with those before revascularization surgery in the $\mathrm{CB}$ group (all $P<.001$ ), which was consistent with the report that the revascularization increased substantive perfusion. ${ }^{31}$ Simultaneously, the postoperative rCBF, rCBV and MTT were also improved after indirect bypass surgery, we inferred that the ingrowth of sticking vessels might also increase the substantive cerebral perfusion in some extent. Although the rTTP was also improved slightly in the IB group, there was no statistical difference in the rTTP as compared with that before revascularization $(P=.067)$. This may be that rTTP reflects the time that the cerebral blood flow reaches a peak rather than the amount of perfusion, and it depends on the diameter of superior vascular lumen, and its delay can't reflect hypoperfusion sufficiently. ${ }^{32}$ Although Wintermark $\mathrm{M}$ et al. showed that rTTP was quite sensitive to the changes of perfusion in the acute period of infarction, the 
follow-up time in this study was $16.5 \pm 8.7$ months, a relatively long time. ${ }^{33}$ Thus, we speculated that the phenomenon was caused by the small new vessels' lumen and small blood flow. In addition, the $\mathrm{CB}$ group's postoperative rCBF was significantly higher than the IB group $(P<.001)$. We speculated that $\mathrm{CB}$ group had better neovascularization receiving dual blood supplies from STA-MCA anatomies and sticking vessels, which were more conducive to improving cerebrovascular autonomic regulation, rapidly increasing parenchymal cerebral perfusion and significantly improving the rCBF.

In summary, combined ameliorating hemodynamic stress in short-term and promoting the neoangiogenesis in long-term, the CB group can perform better neovascularization and improvement of rCBF and AchA-PcoA extension/expansion, which prevented the secondary damage to the neurologic function from recurrent stroke events and got a more satisfactory prognosis. Thus, we inferred that the STA-MCA combined EDAMS is the optimal procedure for hemorrhagic MMD, maybe, and the use of vasoactive and angiogenic medicines locally or systemically is likely to enhance this efficacy, which is well-deserved to be further studied.

\section{Limitations:}

This is a single center and retrospective study. The follow-up time is no long enough. There may exist selection bias in choosing CB or IB revascularization procedure, and the sample size was not large enough with only 68 hemispheres in 37 patients. We failed to consider the reciprocal effects between 2 hemispheres, which remains to be further studied. Although the collateral circulation and hemodynamics in the CB and IB groups were assessed in this study, the factors related to the collateral circulation and hemodynamics were not further analyzed.

\section{Conclusion:}

$\mathrm{CB}$ can obtain better neovascularization, improvement of AchA/PcoA dilation or extension and increased rCBF as compared with IB, as well as lower recurrent ischemic\& hemorrhagic stroke rates and more satisfactory clinical prognosis, which could be the optimal treatment for hemorrhagic MMD.

\section{Abbreviations}

DSA: digital subtraction angiography; CTP: computed tomography perfusion; TTP: time to peak; MTT: mean transit time; CBF: cerebral blood flow; CBV: cerebral blood volume; STA-MCA: superficial temporal artery-to-middle cerebral artery; and EDAMS: encephaloduroarteriomyosynangiosis;PCA: posterior cerebral artery; ACA: anterior cerebral artery $\square$ AchA: Anterior choroidal artery; PcoA: Posterior communicating artery;

\section{Declarations}

Acknowledgements: Not applicable 
Authors' contributions: Jun Sun and Zhang-Yu Li: conception, design, analysis, and interpretation of data. Jun Sun, Zhang-Yu Li and Hao Li: acquisition of data. Jun Sun: drafting the article. Hui Wang, Chuan Chen and Cong Ling: technical support and surgery. Hui Wang: approved the final version of the manuscript on behalf of all authors. Hui Wang and Chuan Chen: study supervision. All authors critically revised the article and reviewed the submitted version of the manuscript.

Funding: This study was supported by the National Natural Science Foundation of China (No.81571202) and Guangdong Provincial Scientific Fundamental Condition Construction (experimental animal science) Project (NO.2019A030317004).

Ethics Approval and consent to participate: All procedures performed in studies involving human participants were in accordance with the ethical standards of the Biomedical Ethics Committee of the Third Affiliated Hospital of Sun Yat-sen University; The informed consent of all patients was obtained.

Consent for publication: Not applicable.

Competing interests: The authors declare that they have no competing interests.

\section{References}

1. Scott RM, Smith ER. Moyamoya disease and moyamoya syndrome. N Engl J Med. 2009;360(12):1226-37.

2. Suzuki J, Takaku A. Cerebrovascular "moyamoya" disease. Disease showing abnormal net-like vessels in base of brain. Arch Neurol. 1969;20(3):288-99.

3. Duan L, Bao XY, Yang WZ, et al. Moyamoya disease in China: its clinical features and outcomes. Stroke. 2012;43(1):56-60.

4. Kim JS. Moyamoya Disease: Epidemiology, Clinical Features, and Diagnosis. J Stroke. 2016;18(1):211.

5. Guzman R, Lee M, Achrol A, et al. Clinical outcome after 450 revascularization procedures for moyamoya disease. Clinical article J Neurosurg. 2009;111(5):927-35.

6. Morioka M, Hamada J, Todaka T, Yano S, Kai Y, Ushio Y. High-risk age for rebleeding in patients with hemorrhagic moyamoya disease: long-term follow-up study. Neurosurgery. 2003;52(5):1049-55.

7. Kang S, Liu X, Zhang D, et al. Natural Course of Moyamoya Disease in Patients with Prior Hemorrhagic Stroke. Stroke. 2019;50(5):1060-6. doi:10.1161/STROKEAHA.118.022771.

8. Kobayashi E, Saeki N, Oishi H, Hirai S, Yamaura A. Long-term natural history of hemorrhagic moyamoya disease in 42 patients. J Neurosurg. 2000;93(6):976-80.

9. Miyamoto S, Yoshimoto T, Hashimoto N, et al. Effects of extracranial-intracranial bypass for patients with hemorrhagic moyamoya disease: results of the Japan Adult Moyamoya Trial. Stroke. 2014;45(5):1415-21. 
10. Jiang $\mathrm{H}, \mathrm{Ni}$ W, Xu B, et al. Outcome in adult patients with hemorrhagic moyamoya disease after combined extracranial-intracranial bypass. J Neurosurg. 2014;121(5):1048-55.

11. Jiang $\mathrm{H}$, Yang $\mathrm{H}$, Ni W, et al. Long-Term Outcomes After Combined Revascularization Surgery in Adult Hemorrhagic Moyamoya Disease. World Neurosurg. 2018;116:e1032-41.

12. Deng $X$, Gao F, Zhang D, et al. Effects of different surgical modalities on the clinical outcome of patients with moyamoya disease: a prospective cohort study. J Neurosurg. 2018;128(5):1327-37.

13. Wang QN, Bao XY, Zhang Y, Zhang Q, Li DS, Duan L. Encephaloduroarteriosynangiosis for hemorrhagic moyamoya disease: long-term outcome of a consecutive series of 95 adult patients from a single center [published online ahead of print, 2018 Jun 1]. J Neurosurg. 2018;1-8.

14. Zhao J, Liu H, Zou Y, Zhang W, He S. Clinical and angiographic outcomes after combined direct and indirect bypass in adult patients with moyamoya disease: A retrospective study of 76 procedures. Exp Ther Med. 2018;15(4):3570-6.

15. Park SE, Kim JS, Park EK, Shim KW, Kim DS. Direct versus indirect revascularization in the treatment of moyamoya disease. J Neurosurg. 2018;129(2):480-9.

16. Huang Z, Ding X, Men W, et al. Clinical features and outcomes in 154 patients with haemorrhagic moyamoya disease: comparison of conservative treatment and surgical revascularization. Neurol Res. 2015;37(10):886-92.

17. Macyszyn L, Attiah M, Ma TS, et al. Direct versus indirect revascularization procedures for moyamoya disease: a comparative effectiveness study. J Neurosurg. 2017;126(5):1523-9.

18. Liu ZW, Han C, Zhao F, et al. Collateral Circulation in Moyamoya Disease: A New Grading System. Stroke. 2019;50(10):2708-15.

19. Matsushima T, Inoue T, Suzuki So, Fujii K, Fukui M, Hasuo K. Surgical treatment of moyamoya disease in pediatric patients-comparison between the results of indirect and direct revascularization procedures. Neurosurgery. 1992;31(3):401-5.

20. Yin H, Liu X, Zhang D, et al. A Novel Staging System to Evaluate Cerebral Hypoperfusion in Patients with Moyamoya Disease. Stroke. 2018;49(12):2837-43.

21. Wintermark M, Reichhart M, Cuisenaire 0 , et al. Comparison of admission perfusion computed tomography and qualitative diffusion- and perfusion-weighted magnetic resonance imaging in acute stroke patients. Stroke. 2002;33(8):2025-31.

22. Lin CJ, Guo WY, Chang FC, et al. Using Flat-Panel Perfusion Imaging to Measure Cerebral Hemodynamics: A Pilot Feasibility Study in Patients with Carotid Stenosis. Med (Baltim). 2016;95(20):e3529.

23. Liu X, Zhang D, Shuo W, Zhao Y, Wang R, Zhao J. Long term outcome after conservative and surgical treatment of haemorrhagic moyamoya disease. J Neurol Neurosurg Psychiatry. 2013;84(3):258-65.

24. Kim JM, Lee SH, Roh JK. Changing ischaemic lesion patterns in adult moyamoya disease. J Neurol Neurosurg Psychiatry. 2009;80(1):36-40. 
25. Yamauchi H, Kudoh T, Sugimoto K, Takahashi M, Kishibe Y, Okazawa H. Pattern of collaterals, type of infarcts, and haemodynamic impairment in carotid artery occlusion. J Neurol Neurosurg Psychiatry. 2004;75(12):1697-701.

26. Kwag HJ, Jeong DW, Lee SH, Kim DH, Kim J. Intracranial hemodynamic changes during adult moyamoya disease progression. J Clin Neurol. 2008;4(2):67-74.

27. Cho WS, Kim JE, Kim CH, Ban SP, Kang HS, Son YJ, et al Cho WS, Kim JE, Kim CH, et al. Long-term outcomes after combined revascularization surgery in adult moyamoya disease. Stroke. 2014;45(10):3025-31.

28. Zhao Y, Yu S, Lu J, et al. Direct Bypass Surgery Vs. Combined Bypass Surgery for Hemorrhagic Moyamoya Disease: A Comparison of Angiographic Outcomes. Front Neurol. 2018;9:1121. Published 2018 Dec 20.

29. Ge P, Ye X, Liu X, et al. Angiographic Outcomes of Direct and Combined Bypass Surgery in Moyamoya Disease. Front Neurol. 2019;10:1267. Published 2019 Dec 3.

30. Morioka M, Hamada J, Todaka T, Yano S, Kai Y, Ushio Y. High-risk age for rebleeding in patients with hemorrhagic moyamoya disease: long-term follow-up study. Neurosurgery. 2003;52(5):1049-55.

31. Zhang J, Wang J, Geng D, Li Y, Song D, Gu Y. Whole-brain CT perfusion and CT angiography assessment of Moyamoya disease before and after surgical revascularization: preliminary study with 256-slice CT. PLoS One. 2013;8(2):e57595.

32. Beaulieu C, de Crespigny A, Tong DC, Moseley ME, Albers GW, Marks MP. Longitudinal magnetic resonance imaging study of perfusion and diffusion in stroke: evolution of lesion volume and correlation with clinical outcome. Ann Neurol. 1999;46(4):568-78.

33. Wintermark $M$, Flanders AE, Velthuis $B$, et al. Perfusion-CT assessment of infarct core and penumbra: receiver operating characteristic curve analysis in 130 patients suspected of acute hemispheric stroke. Stroke. 2006;37(4):979-85.

\section{Figures}




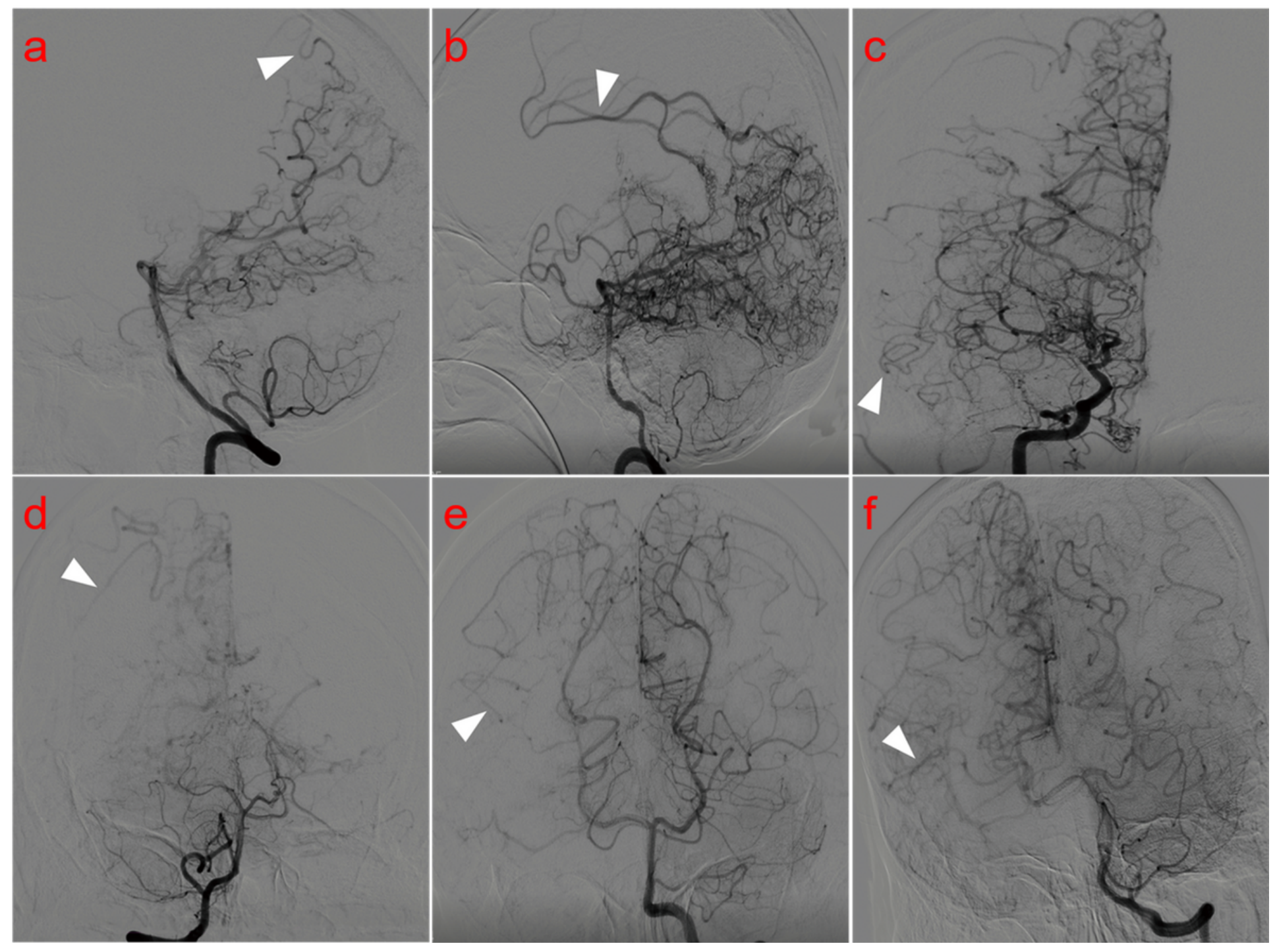

\section{Figure 1}

Preoperative Collateral circulation grading system proposed by Liu et al18 $₫ \mathrm{~A}-\mathrm{B}$ : Leptomeningeal collateral

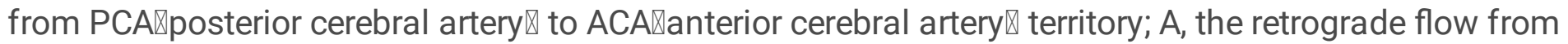
the parietoccipital branches of the PCA to ACA extending to the cortical border zone between the ACA and PCA territory is defined as one score (white arrowhead), and (B) over the central sulcus through the posterior pericallosal artery is defined as 2 scores (white arrowhead). C-F: Leptomeningeal collateral from PCA to ACA territory; C, Anterior temporal PCA branches to temporal MCA territory are assigned with one score. D-F, Parietoccipital PCA branches to the M4(only in superficial vessels), M3(extending into the Sylvian fissure) and M1/2(up to the occlusion) segment of the MCA are assigned with one score $\mathbb{2}$ scores and 3 scores, respectively. In addition, we applied the Suzuki stage to define the expansion of the basal brain perforators in an opposite sequence: Suzuki stage 1 to 6 correspond 1 to 6 to scores. Notes: PCA: posterior cerebral artery; and ACA: anterior cerebral artery 

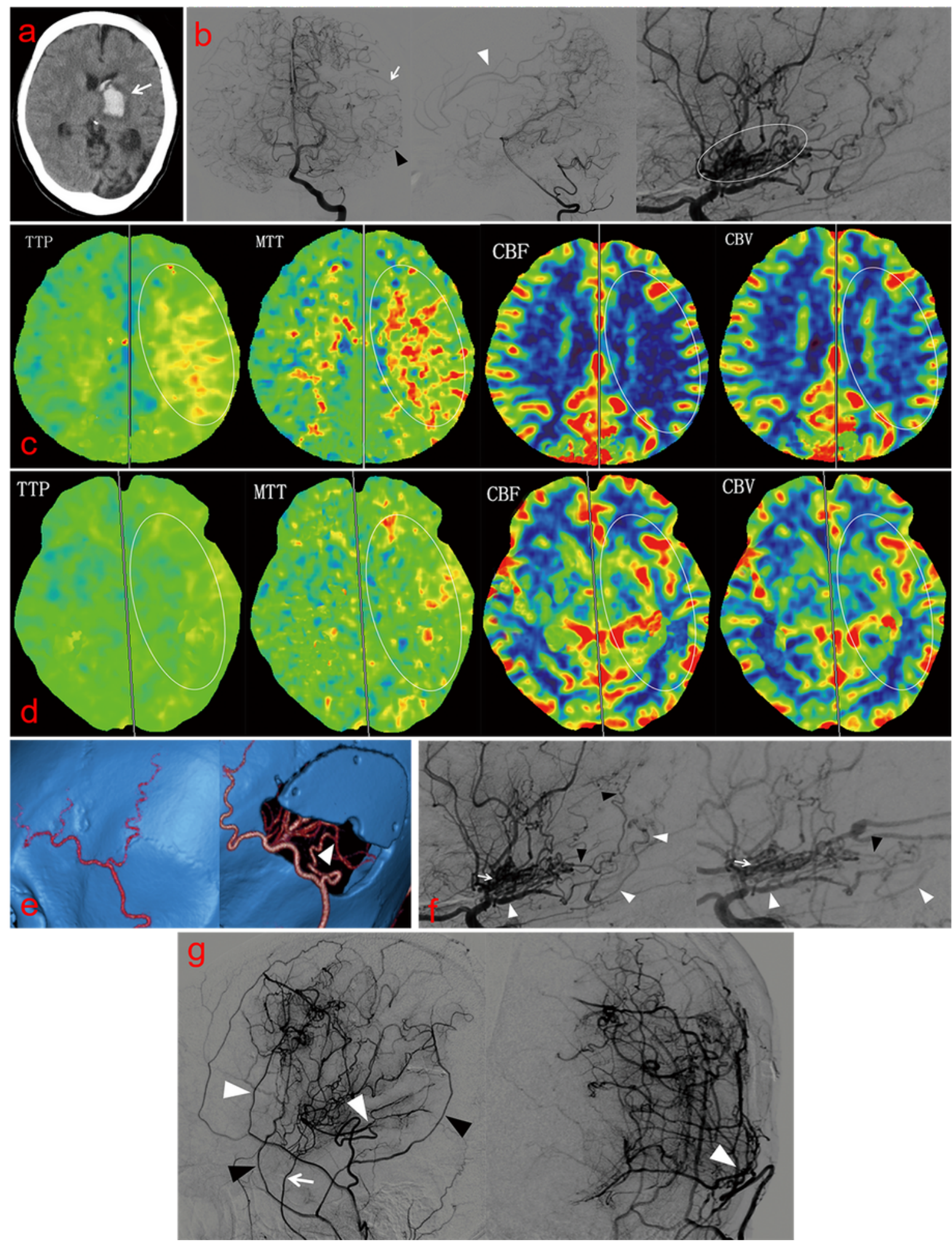

Figure 2

Illustrated case 1: A 43-year-old male patient who underwent CB surgery in the left hemisphere. A, CT images showing the hemorrhage site in the left basal ganglia (white arrow). B, DSA showing that

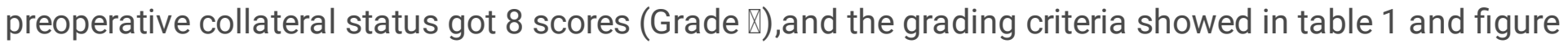
1. C, Representative CTP images showing stage $\otimes$ of preoperative CTP stage the left temporal lobe, and

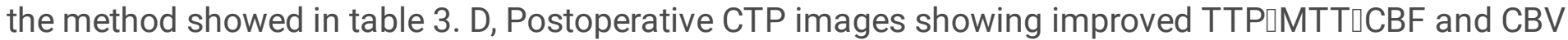


in the left temporal lobe after combined STA-MCA bypass and EDAMS surgery(CB). E, Preoperative and postoperative computed tomography angiographic lateral view showing the course of the graft vessel and STA-MCA anastomosis. F, Preoperative (left) and Postoperative (right) DSA showing improved AchAPcoA dilation and extension (white and black triangles), and the reduction of moyamoya vessels (white arrowhead). G, Anteroposterior and lateral views of DSA showing the establishment of an intracranial and extracranial arterial anastomosis (Matsushima grade A), including STA trunk and STA-MCA anastomosis (white triangles), the middle meningeal artery (black triangles) and deep temporal artery (white arrow).
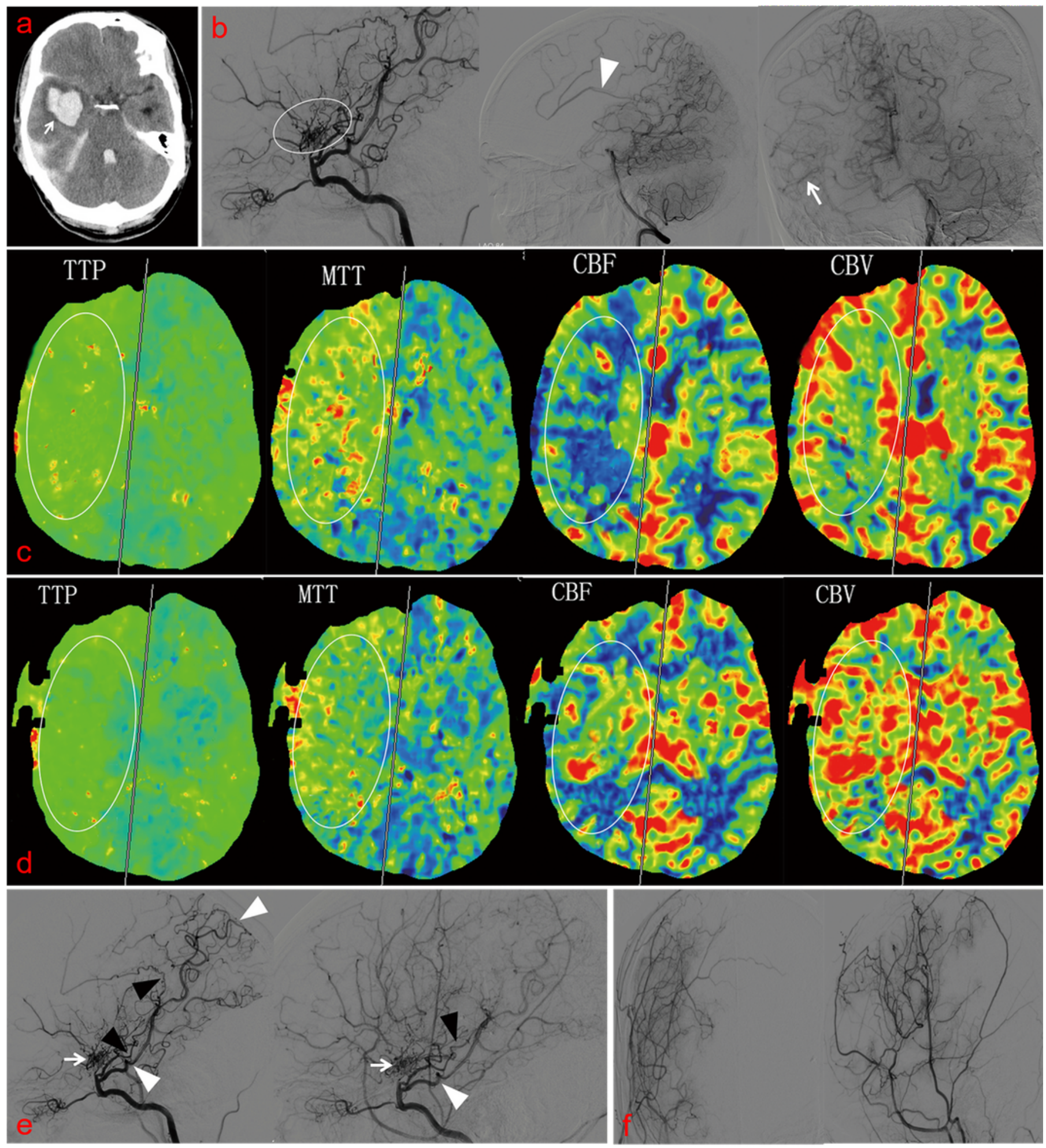


\section{Figure 3}

Illustrated case 2. A 46-year-old female who underwent IB surgery in the right hemisphere. A, CT images showing the hemorrhagic site in the right temporal lobe. B, DSA showing that preoperative collateral status got 6 score (Grade $\nabla$ ). C, Representative CTP images showing stage $\nabla$ of preoperative CTP stage in the right temporal lobe. D, postoperative CTP images showing improved TTP, MTT, CBF and CBV in the left temporal lobe after indirect EDAMS surgery (IB). E, Preoperative (left) and Postoperative (right) of DSA showing improved AchA-PcoA dilation and extension, and the slightly reduction of moyamoya vessels. F, Anteroposterior and lateral views of DSA showing the establishment of neovascularization from intracranial and extracranial arterial anastomosis (Matsushima grade A). 